\title{
ORIGINAL ARTICLE \\ Feasibility of a novel diagnostic chart of intramedullary spinal cord tumors in magnetic resonance imaging
}

\author{
H Arima, T Hasegawa, D Togawa, Y Yamato, S Kobayashi, T Yasuda and Y Matsuyama
}

Study design: Retrospective chart review.

Objectives: Each type of intramedullary spinal cord tumor (IMSCT) has specific anatomical and pathological features visible on magnetic resonance (MR) imaging. The purpose of this study was to investigate the accuracy of preoperative IMSCT diagnosis using our diagnostic chart of tumor-specific MR imaging findings.

Setting: Hamamatsu, Japan.

Methods: From 2009 to 2013, 28 consecutive patients with IMSCT who underwent surgery in our university hospital were included in this study. There were 17 men and 11 women with an average age of 49 years (12-81). The pathological diagnoses were hemangioblastoma (12), ependymoma (11), astrocytoma (4) and squamous cell carcinoma (1). Tumor-specific MR imaging findings were as follows: ependymoma ((a) spinal cord swelling, (b) contrast effect with necrosis, (c) tumor in the center of the spinal cord), hemangioblastoma ((a) spinal cord swelling, (b) homogeneous contrast effect) and astrocytoma ((a) spinal cord swelling, (b) contrast effect is either, (c) eccentric tumor). Based on these features, we generated a diagnostic chart to investigate the MR imaging diagnosis accuracy for IMSCTs.

Results: The accuracy of preoperative diagnosis was $89 \%$ (25/28 cases). Correct diagnoses were made in $100 \%$ of hemangioblastomas (12/12 cases), $90 \%$ of ependymomas ( $9 / 11$ cases) and $100 \%$ of astrocytomas (4/4 cases).

Conclusions: Different types of IMSCTs exhibit unique MR imaging characteristics. These features can be used to preoperatively diagnose IMSCTs with high accuracy.

Spinal Cord (2014) 52, 769-773; doi:10.1038/sc.2014.127; published online 5 August 2014

\section{INTRODUCTION}

Intramedullary spinal cord tumors (IMSCTs) are relatively rare neoplasms, accounting for only $2-4 \%$ of central nervous system glial tumors. $^{1-4}$ The first successful removal of a spinal cord tumor dates back to 1887 and was performed by Gower and Horsley. ${ }^{5}$ At that time, diagnosis and preoperative planning consisted solely of a detailed neurological examination for precise lesion localization., ${ }^{5,6}$ The introduction of modern imaging techniques, such as magnetic resonance (MR) imaging, has greatly improved the diagnosis of spinal cord lesions. ${ }^{7-11}$ The ability to preoperatively predict pathological diagnoses could considerably enhance surgical planning and the ability of the surgeon to describe the procedure to patients and their families. However, the frequency of IMSCT is low, and these types of tumors exhibit a variety of MR imaging findings. ${ }^{7-9}$ In addition, characteristic imaging findings that can aid in diagnoses have not yet been identified. This makes some cases of IMSCT difficult to diagnose. We established a diagnostic chart according to the tumor-specific MR imaging findings. The purpose of this study was to investigate the accuracy of MR imaging for IMSCT using this diagnostic chart.

\section{MATERIALS AND METHODS}

\section{Patient population}

The study design was a retrospective chart review. From December 2009 to July 2013, 28 cases of IMSCT were treated in our university hospital. There were 17 male and 11 female patients with an average age of 49. Pathological diagnoses were ependymoma, hemangioblastoma, astrocytoma and squamous cell carcinoma in 12,11, 4 and 1 cases, respectively. All patients underwent 1.5-T MR imaging, and we examined T1- and T2-weighted images and gadolinium-enhanced contrast images.

\section{MR imaging findings}

MR images were investigated with special reference to spinal cord swelling, high-signal intensity on T2-weighted images, contrast effect and tumor location on axial view.

Spinal cord swelling. We examined whether or not there was spinal cord swelling in sagittal T2-weighted images.

High-signal intensity on T2-weighted images. We assessed the presence of high-signal areas in sagittal or axial T2-weighted images.

Contrast effect. We examined the effect of increased tumor substance with gadolinium-enhanced images. If there was a contrast effect, we determined whether or not it was homogeneous.

Tumor localization on axial images. We examined tumor localization in contrast axial images to understand the relationship between the tumor and spinal cord and to determine if the tumor was central or eccentric.

Analysis of the possibility of IMSCT diagnosis by MR imaging Based on these features, we established an IMSCT diagnostic chart for diagnosis. We investigated the accuracy of MR imaging diagnosis for IMSCT using this diagnostic chart. 


\section{RESULTS}

\section{MR imaging findings}

Spinal cord swelling. Spinal cord swelling was seen in all cases but varied from focal to diffuse.

High-signal intensity on T2-weighted images. Compared to the signal of the normal spinal cord, high-signal areas in sagittal or axial T2weighted images were observed in all cases. In 2 out of 11 cases of ependymoma, high brightness was observed compared to the normal spinal cord, but it was not brighter than cerebrospinal fluid.

Contrast effect. A contrast effect was noted in all cases of ependymoma, hemangioblastoma and squamous cell carcinoma, and in three out of four astrocytoma cases (Figure 1). Ependymoma cases exhibited a contrast effect with a necrotic region (Figure 2). Hemangioblastoma imaging exhibited a homogenous strong contrast effect

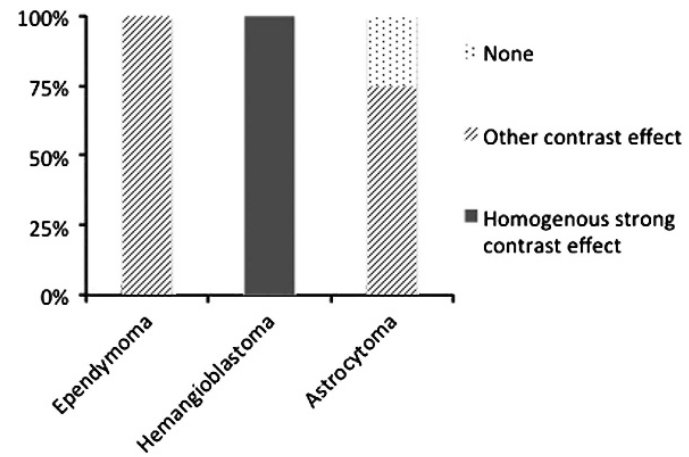

Figure 1 Histogram showing the contrast effect in each type of intramedullary spinal cord tumor. Contrast effect was seen in all cases of hemangioblastoma and ependymoma, and in three out four astrocytoma cases. All hemangioblastomas showed homogenous strong contrast effects, but ependymoma and astrocytoma cases did not.
(Figure 3). All, except one, cases of astrocytoma showed contrast effects with unclear margins (Figure 4).

Tumor localization in axial images. All ependymoma and hemangioblastoma cases were central and eccentric type, respectively(Figure 5). One astrocytoma case was central type, two were eccentric and another case was uncharacterizable because there was no contrast effect. One squamous cell carcinoma was eccentric.

We established an IMSCT diagnostic chart based on the MR imaging features (Figure 6). First, we assessed spinal cord swelling and high-signal intensity on T2-weighted image, followed by the contrast effect. When there was no contrast effect, a tumor was classified as astrocytoma. We considered a tumor to be hemangioblastoma when there was a uniform strong contrast effect. Next, we assessed tumor localization. Central-type tumors were considered as ependymomas and eccentric tumors were considered as astrocytomas.

When our chart was used retrospectively, the accuracy of MR imaging diagnosis was $89 \%$ ( 25 out of 28 cases). In looking at each tumor type, 9/11 ependymoma cases matched (82\%), all hemangioblastoma cases matched (100\%) and 4/4 astrocytoma cases matched (100\%) (Figure 7).

\section{DISCUSSION}

IMSCTs account for $2-4 \%$ of all central nervous system tumors ${ }^{1-4}$ and the most frequent diagnoses are ependymoma, hemangioblastoma and astrocytoma. IMSCT should be surgically treated as soon as neurological symptoms appear. ${ }^{12}$ Gross total resection is attempted for the majority of benign pathologies; ${ }^{2,3,4,13}$ however, some astrocytoma cases are malignant and it has a greater neurological risk to perform gross total resection in these patients. ${ }^{4,10,13,14}$ When we design a surgical plan for IMSCT, it is desirable to predict the appropriate tissue diagnosis, although a biopsy is required and involves preparation equivalent to resection. There is also the possibility that nerves can be damaged during surgery. It would be very useful if we could predict the histological
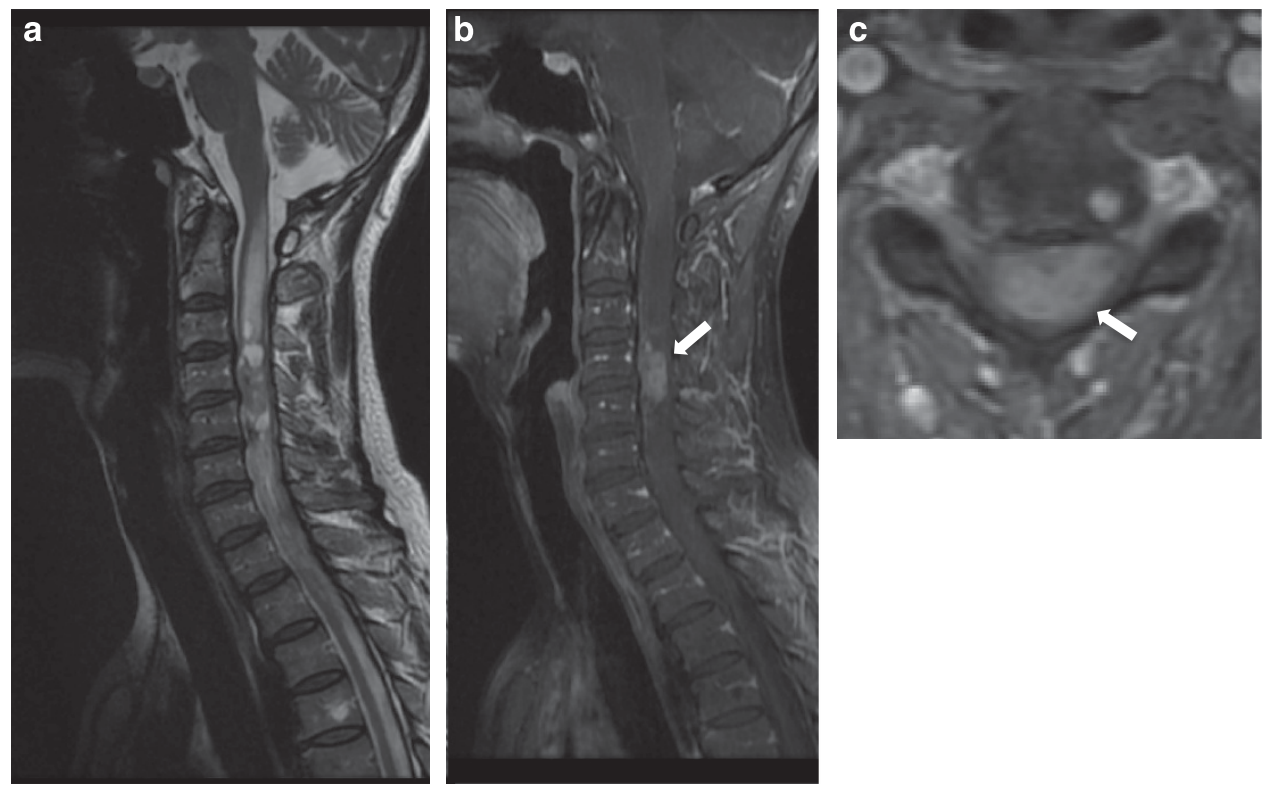

Figure 2 Magnetic resonance image showing an ependymoma in a 55-year-old female. (a) T2-weighted image, sagittal view. (b) Contrast image, sagittal view. (c) Contrast image, axial view. A heterogeneous contrast effect is observed (arrow). 

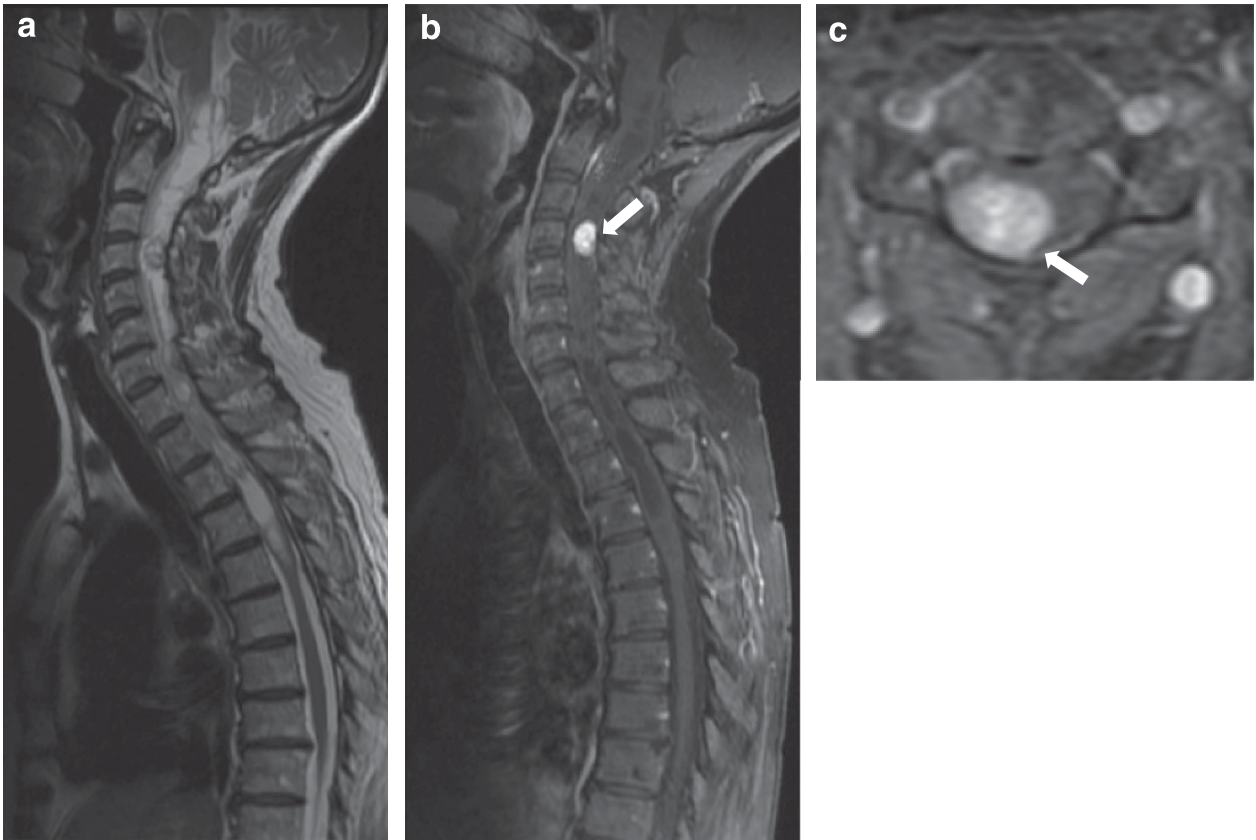

Figure 3 Magnetic resonance image showing a hemangioblastoma in a 64-year-old female. (a) T2-weighted image, sagittal view. (b) Contrast image, sagittal view. (c) Contrast image, axial view. A homogenous strong contrast effect is observed (arrow).
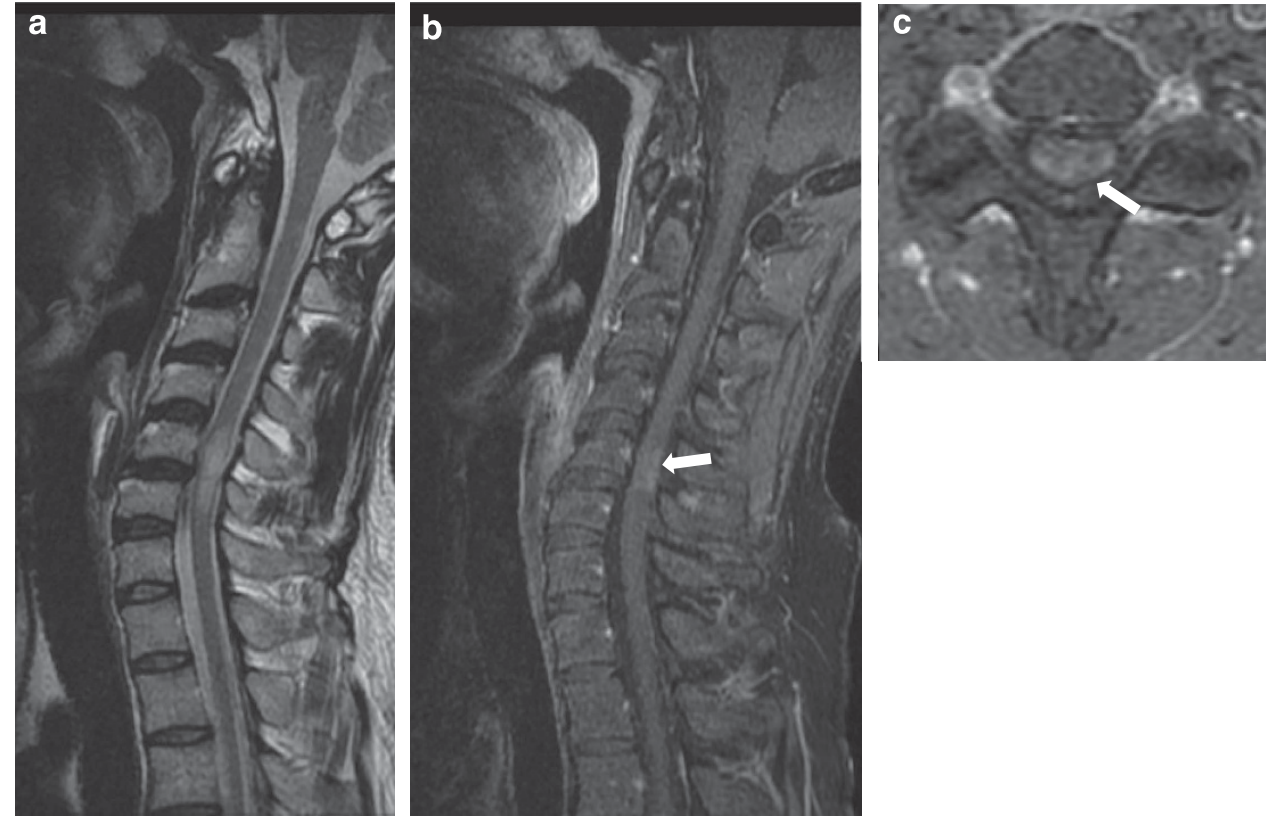

Figure 4 Magnetic resonance image showing an astrocytoma in a 38-year-old male. (a) T2-weighted image, sagittal view. (b) Contrast image, sagittal view. (c) Contrast image, axial view. A contrast effects with unclear margins is observed (arrow).

diagnosis preoperatively by MR imaging findings. Because MR images of IMSCT vary, it is necessary to carefully examine the features..$^{7-11}$

We focused on spinal cord swelling, high-signal intensity on a T2-weighted image, contrast effect and tumor location on axial images as criteria for MR imaging-based diagnosis. It was previously reported that IMSCT almost always manifests as spinal cord swelling. ${ }^{9,15}$ In our study, spinal cord swelling was seen in all cases. In addition, compared to the normal spinal cord signal, we observed high-signal areas in sagittal or axial T2-weighted images in all cases. Therefore, in our chart, we first evaluate spinal cord swelling and the presence of high-signal intensity on T2-weighted images. The vast majority of IMSCTs show at least some contrast effect following the intravenous administration of gadolinium-based contrast material. ${ }^{6-8}$ There was a contrast effect in 27 out of 28 cases in our study. Ependymomas tend to be strongly but heterogeneously enhanced and often have well-defined margins. ${ }^{7,9,12,16}$ In hemangioblastoma, 


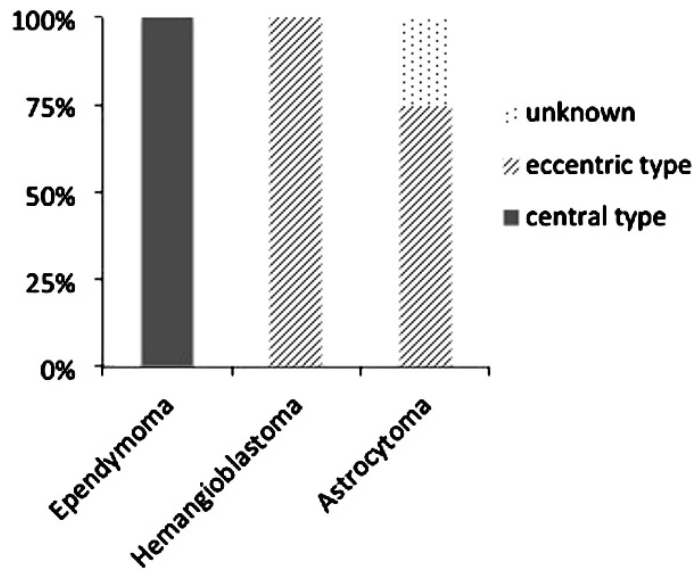

Figure 5 Histogram showing tumor localization on axial images. All ependymomas were central type. For hemangioblastoma, five and six cases were central and eccentric types, respectively. For astrocytoma, two cases were central type, and two were eccentric type.

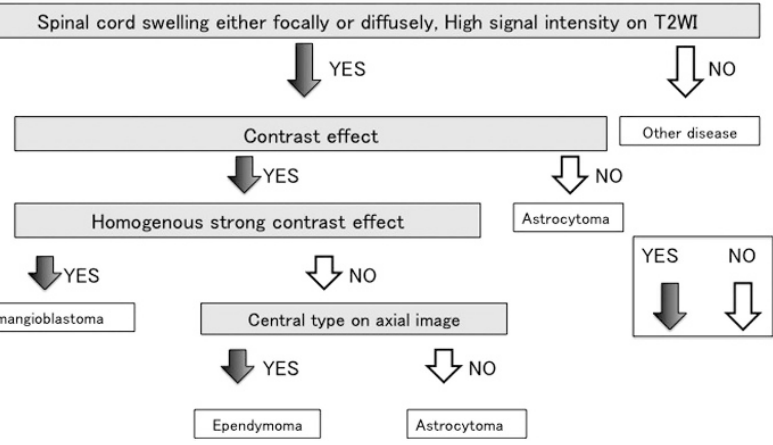

Figure 6 Schematic illustration of the intramedullary spinal cord tumor diagnostic chart based on MR imaging features.

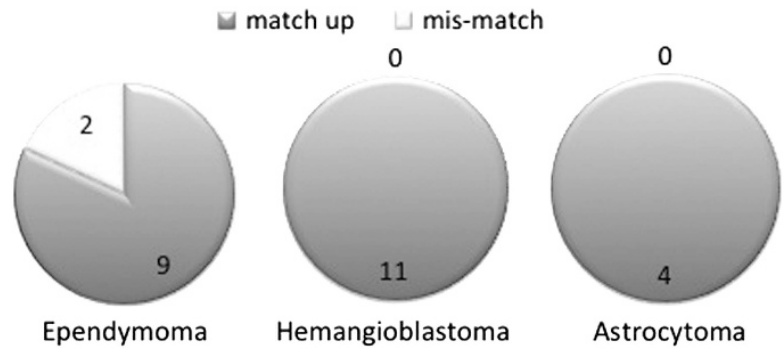

Figure 7 Pie chart showing match and mismatch for each type of intramedullary spinal cord tumor. For ependymoma, 9 out of 11 cases matched (88\%). All hemangioblastoma cases matched (100\%). All astrocytoma cases matched (100\%).

contrast-enhanced MR images typically show an intense, homogenously enhanced subpial tumor nodule, allowing differentiation of the small nidus from the adjacent edematous cord and peritumoral cyst, as well as from arteriovenous fistulae, cavernomas and other hypervascular IMSCTs. ${ }^{7,9}$ Astrocytomas almost always enhance immediately when contrast material is administered. However, enhancement can be heterogeneous because the lesion margins are often poorly defined and irregular. There are some reports on localization differences for ependymomas and astrocytomas on axial images. ${ }^{7,9,16}$ It was reported that ependymomas tend to be central, whereas astrocytomas arise eccentrically within the posterior cord. ${ }^{7,9,16}$ The results of our study confirm that ependymomas tend to be central, whereas hemangioblastomas and astrocytomas are eccentric. Epstein et al. ${ }^{15}$ hypothesized that ependymomas are related to the origin of tumor around the central canal and they seem to expand symmetrically and circumferentially, compressing or interrupting crossing spinothalamic tracts.

In the present study, we established a diagnostic chart according to these tumor-specific MRI findings. According to Fisher's report, the frequency of ependymoma, hemangioblastoma and astrocytoma is $\sim 75 \% .{ }^{17}$ Using our diagnostic chart, we believe that most IMSCTs could be accurately diagnosed. The diagnosis rate was $89 \%$ in the present study. Previously, Ozawa et al. ${ }^{18}$ attempted to diagnose IMSCT using MR imaging. They focused on tumor size, tumor localization in the transverse image, T1 and T2 signal intensity, lowsignal area of the peripheral and internal tumor in $\mathrm{T} 2$-weighted images, contrast effect of the tumor and syrinx formation as the criteria for MR imaging-based diagnosis. They also created an IMSCT diagnostic chart and reported a success rate of $79 \%$. We simplified their chart and narrowed the targets to the most frequent IMSCTs: ependymoma, hemangioblastoma and astrocytoma. The new chart showed a good diagnostic rate. Non-specialists for spinal cord tumors may not be familiar with IMSCTs, which are relatively rare; we think that this chart will be useful for a non-specialist of spinal cord tumor and the training physician. However, needless to say, it is still important to submit tumor tissues for pathologic diagnosis during and after the surgery.

\section{Limitation}

There are several limitations in this study. The first is that the number of cases is small. Second is that the group that is a source of this chart and the group to which we did validation studies are the same. In future, we will examine the effectiveness of this chart by applying to the other IMSCT patients.

\section{CONCLUSION}

We established an IMSCT diagnostic chart based on the MR imaging features of ependymoma, hemangioblastoma, and astrocytoma. This chart enables surgeons to preoperatively diagnose IMSCT with high accuracy.

\section{DATA ARCHIVING}

There were no data to deposit.

\section{CONFLICT OF INTEREST}

The authors declare no conflict of interest.

1 Brotchi J. Intrinsic spinal cord tumor resection. Neurosurgery 2002; 50: 1059-1063.

2 Constantini S, Miller DC, Allen JC, Rorke LB, Freed D, Epstein FJ. Radical excision of intramedullary spinal cord tumors: surgical morbidity and long-term follow-up evaluation in 164 children and young adults. J Neurosurg 2000; 93: 183-193.

3 Kzane PJ, el-Mahdy W, Singh A, Powell MP, Crockard HA. Spinal intradural tumours: Part II-Intramedullary. Br J Neurosurg 1999; 13: 558-563.

4 Shrivastava RK, Epstein FJ, Perin NI, Post KD, Jallo GI. Intramedullary spinal cord tumors in patients older than 50 years of age: management and outcome analysis. J Neurosurg Spine 2005; 2: 249-255. 
5 Manzano G, Green BA, Vanni S, Levi AD. Contemporary management of adult intramedullary spinal tumors-pathology and neurological outcomes related to surgical resection. Spinal Cord 2008; 46: 540-546.

6 Regelsberger J, Fritzsche E, Langer N, Westphal M. Intraoperative sonography of intraand extramedullary tumors. Ultrasound Med Biol 2005; 31: 593-598.

7 Do-Dai DD, Brooks MK, Goldkamp A, Erbay S, Bhadelia RA. Magnetic resonance imaging of intramedullary spinal cord lesions: a pictorial review. Curr Probl Diagn Radiol 2010; 39: 160-185.

8 Goy AM, Pinto RS, Raghavendra BN, Epstein FJ, Kricheff II. Intramedullary spinal cord tumors: MR imaging, with emphasis on associated cysts. Radiology 1986; 161: $381-386$.

9 Koeller KK, Rosenblum RS, Morrison AL. Neoplasms of the spinal cord and filum terminale: radiologic-pathologic correlation. Radiographics 2000; 20 : 1721-1749.

10 Sandalcioglu IE, Gasser T, Asgari S, Lazorisak A, Engelhorn T, Egelhof T et al. Functional outcome after surgical treatment of intramedullary spinal cord tumors: experience with 78 patients. Spinal Cord 2005; 43: 34-41.
11 van Velthoven V. Intraoperative ultrasound imaging: comparison of pathomorphological findings in US versus CT, MRI and intraoperative findings. Acta Neurochir Suppl 2003; 85: 95-99.

12 McCormick PC, Torres R, Post KD, Stein BM. Intramedullary ependymoma of the spinal cord. J Neurosurg 1990; 72: 523-532.

13 Klekamp J. Treatment of intramedullary tumors: analysis of surgical morbidity and long-term results. J Neurosurg Spine 2013; 19: 12-26.

14 Raco A, Esposito V, Lenzi J, Piccirilli M, Delfini R, Cantore G. Long-term follow-up of intramedullary spinal cord tumors: a series of 202 cases. Neurosurgery 2005; 56: 972-981.

15 Epstein FJ, Farmer JP, Freed D. Adult intramedullary spinal cord ependymomas: the result of surgery in 38 patients. J Neurosurg 1993; 79: 204-209.

16 Miller DC. Surgical pathology of intramedullary spinal cord neoplasms. J Neurooncol 2000; 47: 189-194.

17 Fischer G, Brotchi J. Intramedullary Spinal Cord Tumors. Thieme: Stuttgart, 1996.

18 Ozawa H, Sato T, Kokubun S. Differential diagnosis of intramedullary spinal cord tumors by MRI. Orthop Surg Traumatol 2001; 44: 273-278. 